\title{
Reciprocal neural response within lateral and ventral medial prefrontal cortex during hot and cold reasoning
}

\author{
Vinod Goel ${ }^{\mathrm{a}, \mathrm{b}, *}$ and Raymond J. Dolan ${ }^{\mathrm{a}}$ \\ ${ }^{a}$ Wellcome Department of Cognitive Neurology, Institute of Neurology, Queens Square, London WC1N $3 B G$, UK \\ ${ }^{\mathrm{b}}$ Department of Psychology, York University, Toronto, Ontario M3J 1P3, Canada
}

Received 21 April 2003; revised 27 July 2003; accepted 31 July 2003

\begin{abstract}
Logic is widely considered the basis of rationality. Logical choices, however, are often influenced by emotional responses, sometimes to our detriment, sometimes to our advantage. To understand the neural basis of emotionally neutral ("cold") and emotionally salient ("hot") reasoning we studied 19 volunteers using event-related fMRI, as they made logical judgments about arguments that varied in emotional saliency. Despite identical logical form and content categories across "hot" and "cold" reasoning conditions, lateral and ventral medial prefrontal cortex showed reciprocal response patterns as a function of emotional saliency of content. "Cold" reasoning trials resulted in enhanced activity in lateral/dorsal lateral prefrontal cortex (L/DLPFC) and suppression of activity in ventral medial prefrontal cortex (VMPFC). By contrast, "hot" reasoning trials resulted in enhanced activation in VMPFC and suppression of activation in L/DLPFC. This reciprocal engagement of L/DLPFC and VMPFC provides evidence for a dynamic neural system for reasoning, the configuration of which is strongly influenced by emotional saliency.
\end{abstract}

(C) 2003 Elsevier Inc. All rights reserved.

\section{Introduction}

Rational and emotional behavior are often seen to be in opposition, with the former linked to reason and logic, and much of what is uniquely human, and the latter connected to "animal passions" (Henle, 1962). This dichotomy is captured by phrases such as "using your head" versus "making a gut response." Rational behavior is goal-oriented and widely described as "careful," "calculating," and "evaluating," whereas emotional responses are described as "passionate," "rash," and "acting in the heat of the moment." Despite this apparent separation, the rational and emotional coexist in the real world, and most people experience situations where rational choices are preempted or muddied by emotional biases.

Much neuropsychological data implicate ventral medial prefrontal cortex (VMPFC) in emotional processing (Damasio, 1997; Goel and Dolan, 2001a; Harlow, 1868;

* Corresponding author. Fax: +416-736-5814.

E-mail address: vgoel@yorku.ca (V. Goel).
Lane et al., 1997; Pietrini et al., 2000) and the lateral/ dorsolateral prefrontal cortex (L/DLPFC) in high-level cognitive processing (Drewe, 1974; Goel et al., 1997; Goldman-Rakic, 1987; Rowe et al., 2001; Shallice, 1988; Stuss and Levine, 2002), including logical reasoning (Goel et al., 1997, 1998, 2000; Goel and Dolan, 2001b, 2003; Houde et al., 2001; Knauff et al., 2002; Parsons and Osherson, 2001). However, with a few exceptions (Gray et al., 2002), the literatures on cognition and emotion are largely independent of each other. It has been observed that some patients with VMPFC lesions, in addition to having significant emotional and social problems, make poor decisions in real-world situations (Anderson et al., 2000). This has led to the suggestion that there is a causal connection between emotional deficits and poor decision-making (Bechara et al., 2000; Damasio, 1996). One possible basis for this connection is an interaction between L/DLPFC and VMPFC during reasoning. While several neuroimaging and patient studies have shown a dissociation between L/DLPFC and VMPFC in cognitive and affective processing (Goel and Dolan, 2001a; Greene et al., 2001; Hariri et al., 2000; Koechlin et al., 2000; Stuss and Levine, 2002), a predicted interaction be- 
Emotional Content

Reasoning Some wars are not unjustified.
All wars involve raping of women.
Some raping of women is not unjustified.

Baseline
Some wars are not unjustified. All wars involve raping of women. Some Indians are dishonest.
Neutral Content

Some Canadians are not children

All Canadians are people

Some people are not children

Some Canadians are not children All Canadians are people Some babies are curious.

\section{a}

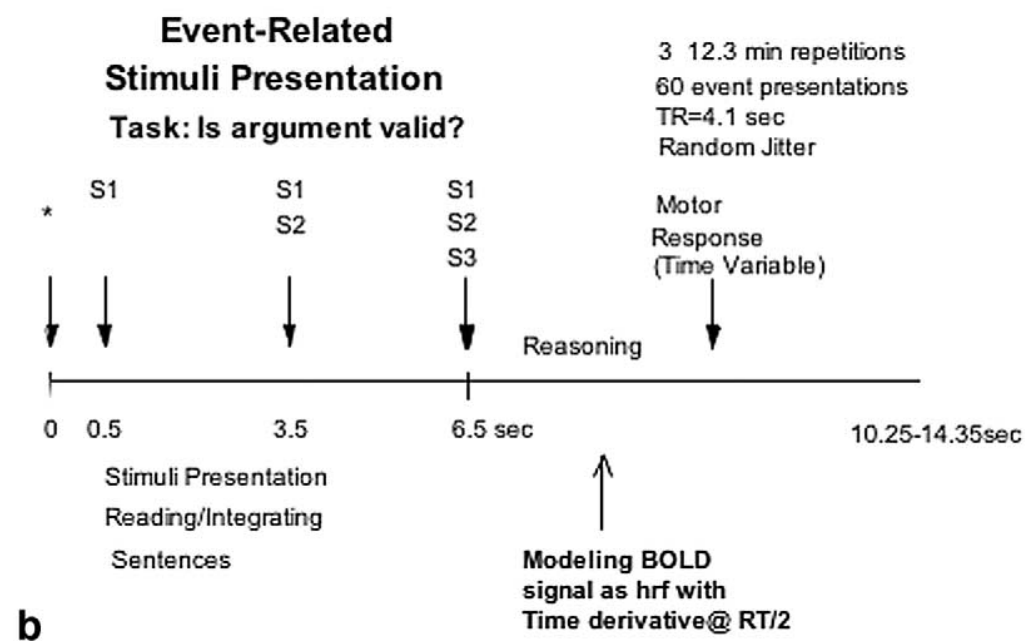

Fig. 1. (a) Experimental design and sample stimuli. (b) Specifics of stimuli presentation.

tween L/DLPFC and VMPFC in reasoning tasks, as a function of emotional saliency, remains to be demonstrated.

To test the hypothesis of interaction between L/DLPFC and VMPFC during rational thought, we scanned 19 righthanded normal subjects using event-related fMRI, while they engaged in identical logical reasoning tasks that varied only in emotional saliency. After the scanning session subjects reviewed and rated each item for saliency of content. Our findings indicate that the neural systems engaged by logical reasoning are dynamically configured as a function of the emotional saliency of the content. Specifically, we show a reciprocal response in L/DLPFC and VMPFC that reflects the degree to which reasoning is "cold" or "hot."

\section{Method}

\section{Subjects}

Nineteen right-handed normal subjects (7 male and 12 female), with a mean age of 32.4 years $(S D=10.5)$ and mean education level of 16.2 years $(\mathrm{SD}=1.6)$, volunteered to participate in the study. All subjects gave informed consent and the study was approved by the Joint National Hospital for Neurology and Neurosurgery/Institute of Neurology Ethics Committee.

\section{Experimental design}

Sixty emotionally salient and 60 emotionally neutral syllogisms, encompassing 24 different forms ${ }^{1}$ were organized into a $2 \times 2$ factorial design (Fig. 1a). The first factor was Content, in which half the arguments contained sentences that subjects could be expected to consider emotionally neutral, while the other half contained sentences that subjects could be expected to consider emotionally salient or charged. This allocation of trials was confirmed by subjects' postscan ratings of saliency of all items (see below). The logically relevant information in both conditions was identical and the key terms were matched for category (living things, objects, abstract ideas). The conditions were also balanced for congruent and incongruent trials. (Congruent trials are either valid arguments with a true (or believable) conclusion or invalid arguments with a false (or unbelievable) conclusion. Incongruent trials are either invalid arguments with a true conclusion or valid arguments with a false conclusion.) Sample arguments are presented in Table 1. The second factor was Task in which the first level

\footnotetext{
${ }^{1}$ The following forms of syllogisms (encoded as per Evans et al., 1993b): were utilized: AA1, AA3, AA4, AE2, AE4, AI1, AI3, AO2, EA1, EA2, EI1, EI2, EI3, EI4, E04, IA1, IA3, IA4, IE1, II3, II4, OA3, OE1, OI4. Some valid forms also had invalid "counterparts."
} 
Table 1

Sample arguments from emotionally salient and neutral categories

All murderous people are criminals.

All Nazis were murderous.

Some Nazis are criminals.

All child molesters are perverse.

Some child molesters are priests.

Some priests are perverse.

Some marathon runners are smokers. All marathon runners are healthy.

Some smokers are healthy.

No one with a criminal record is innocent.

Some pedophiles have criminal records.

Some pedophiles are not innocent.

Some blacks are pimps.

All pimps are criminals.

Some blacks are not criminals.

No gay people are perverse.

Some priests are perverse.

Some priests are gay.

All handicapped are capable.

All amputees are handicapped.

Some amputees are not capable.

All Chinese are Oriental.

Some Chinese are not smart.

Some Orientals are not smart.

No doctors are criminals.

Some doctors are rapists.

Some rapists are not criminals.

Some kids are not expendable.

No disabled people are kids.

Some disabled people are expendable.
Some policemen are detectives.

All detectives are competent.

Some policemen are competent.

No poisons are sold at the grocers.

Some mushrooms are sold at the grocers.

Some mushrooms are not poisonous.

All gossip rags are popular.

All Hello magazines are gossip rags.

All Hello magazines are popular.

All African animals can be obedient.

Some African animals are fierce.

Some fierce animals can be obedient.

Some rock stars are guitarists.

All guitarists can sing.

Some rock stars cannot sing.

No reptiles can grow hair.

Some elephants can grow hair.

No elephants are reptiles.

All French poodles are dogs.

Some French poodles are small.

Some small animals are not dogs.

Some mammals are donkeys.

All mammals are animals.

Some animals are not donkeys.

No Catholic nuns are priests.

Some women are Catholic nuns.

No priests are women.

No mutes are opera singers.

Some musicians are mute.

Some musicians are not opera singers. (a reasoning condition) involved stimuli that constituted legitimate arguments, half of which were valid while the other half were invalid. The second-level (baseline condition) trials were generated by taking the above arguments and switching around the third sentence such that the three sentences did not constitute arguments. There were 20 neutral and 20 emotional baseline trials and 20 "rest" trials where subjects simply viewed a fixation cross. Sentences in all trials were grammatical, meaningful, and matched for length across conditions.

After the scan subjects rated each trial for salience of content using a published scale (Bradley and Lang, 1994). Subjects were asked "How does this item make you feel?" The scale described high salience items as those that made subjects feel "stimulated," "tense," and "excited," while low salience items were those that made subjects feel "relaxed," "calm," and "dull." The scale ranged from 5+ to 5-, with zero signifying neutral.

\section{Stimuli presentation}

Stimuli from all conditions were presented randomly in an event-related design (Fig. 1b; the asterisk indicates the start of a trial at $0 \mathrm{~s}$ ). The sentences appeared on the screen one at a time with the first sentence appearing at $500 \mathrm{~ms}$, the second at $3500 \mathrm{~ms}$, and the last sentence at $6500 \mathrm{~ms}$. All sentences remained on the screen until the end of the trial. The length of trials varied from 10.25 to $14.35 \mathrm{~s}$, leaving subjects 3.75 to $7.85 \mathrm{~s}$ to respond. The task in all conditions was the same. Subjects were required to determine whether the conclusion followed logically from the premises (i.e., whether the argument was valid). Subjects responded by pressing a button on a keypad after the appearance of the last sentence. In (reasoning condition) trials where the three sentences constituted an argument, subjects had to determine the validity of the argument. In (baseline) trials, where the first two sentences were related, subjects would begin to construct a representation of the problem, but when the third, unrelated, sentence appeared it would not be integrated into a coherent representation (i.e., search for a solution) and subjects would respond "no." Subjects were instructed to respond as quickly as possible and move to the next trial if the stimuli advanced before they could respond. Subjects reviewed example stimuli from each condition prior to being scanned to ensure that they understood the task 


\section{fMRI scanning technique}

A 2-T Siemens VISION system (Siemens, Erlangen, Germany) was used to acquire T1 anatomical volume images $(1 \times 1 \times 1.5$-mm voxels $)$ and $48 \mathrm{~T} 2 *$-weighted echoplanar images $(64 \times 643 \times 3-\mathrm{mm}$ pixels, $\mathrm{TE}=40 \mathrm{~ms})$ sensitive to blood oxygenation level dependent (BOLD) contrast; 1.8-mm-thick echoplanar images were acquired axially every $3 \mathrm{~mm}$, positioned to cover the whole brain..$^{2}$ Data were recorded during a single acquisition period. A total of 558 volume images were acquired over three sessions (186 volumes per session) with a repetition time (TR) of $4.1 \mathrm{~s} / \mathrm{vol}-$ ume. The first 6 volumes in each session were discarded (leaving 180 volumes per session) to allow for T1 equilibration effects. Trials from all conditions were randomly presented in a single-event design. The mean trial time was $12300 \mathrm{~ms} \pm 2050 \mathrm{~ms}$ (TR) with a random jitter. Trials thus varied from 10.25 to $14.35 \mathrm{~s}$. There were 60 event presentations during a session for a total of 180 over the three sessions. Each session lasted $12.3 \mathrm{~min}$. The scanner was synchronized with the presentation of all trials in each session.

\section{Data analysis}

Data were analyzed using Statistical Parametric Mapping (SPM 99) (Friston et al., 1995). All volumes were spatially realigned to the first volume (head movement was $<2 \mathrm{~mm}$ in all cases) and temporally realigned to the AC-PC slice, to account for different sampling times of different slices. A mean image created from the realigned volumes was coregistered with the structural T1 volume and the structural volumes were spatially normalized to the Montreal Neurological Institute brain template (Evans et al., 1993a) using nonlinear basis functions (Ashburner and Friston, 1999). The derived spatial transformation was then applied to the realigned $\mathrm{T} 2 *$ volumes, which were finally spatially smoothed with a 12-mm FWHM isotropic Gaussian kernel (in order to make comparisons across subjects and to permit application of random field theory for corrected statistical inference (Worsley and Friston, 1995)). The resulting time series across each voxel were high-pass-filtered with a cutoff of $32 \mathrm{~s}$, using cosine functions to remove section-specific low-frequency drifts in the BOLD signal. Global means were normalized by proportional scaling to a grand mean of 100 , and the time series temporally smoothed with a canonical hemodynamic response function to swamp small temporal autocorrelations with a known filter.

The BOLD signal was modeled as a hemodynamic response function with time derivative at the midway point between the presentation of the third sentence and the motor response (on a subject by subject, trial by trial basis). The

\footnotetext{
${ }^{2}$ The thin 1.8-mm slices, with 1.2-mm gap, and a relatively short echo time of $40 \mathrm{~ms}$ serve to minimize dropout and distortion (Deichmann et al., 2002; Gorno-Tempini et al., 2002; Hutton et al., 2002).
}

Table 2

Behavioural performance scores and reaction times on task

\begin{tabular}{|c|c|c|c|c|}
\hline & \multicolumn{2}{|c|}{ Performance scores (SD) } & \multicolumn{2}{|l|}{ Rts (SD) } \\
\hline & $\begin{array}{l}\text { Emotional } \\
(\%)\end{array}$ & $\begin{array}{l}\text { Neutral } \\
(\%)\end{array}$ & $\begin{array}{l}\text { Emotional } \\
(\mathrm{ms})\end{array}$ & $\begin{array}{l}\text { Neutral } \\
(\mathrm{ms})\end{array}$ \\
\hline Reasoning trials & $64.5(6.2)$ & $65.9(8.4)$ & 3497 (538) & $3606(516)$ \\
\hline Baseline trials & $98.0(3.8)$ & $98.1(4.9)$ & $1665(403)$ & $1594(416)$ \\
\hline
\end{tabular}

presentation of all three sentences, along with the motor response, was modeled out in the analysis. Condition effects at each voxel were estimated according to the general linear model and regionally specific effects compared using linear contrasts. Each contrast produced a statistical parametric map of the $t$ statistic for each voxel, which was subsequently transformed to a unit normal $Z$ distribution. We report all activations surviving voxel-level intensity threshold of $P<$ 0.05 (corrected for multiple comparisons in a random effect model using False Discovery Rate) (Genovese et al., 2002) and a cluster level threshold of $P<0.05$.

\section{Results}

Behavioral scores indicated that subjects performed the task in the expected manner (Table 2). Subjects' ratings of emotional saliency of content were significantly higher for the emotionally charged material $(+0.82, \mathrm{SD}=0.74)$ than the emotionally neutral material $(-0.72, \mathrm{SD}=0.58)(t(157)$ $=14.62, P<0.0001)$, indicating that the stimuli categories were experienced in the expected direction. There were no differences in subjects' performance on the emotionally neutral and emotionally salient arguments. Subjects took a mean of $3606 \mathrm{~ms}(\mathrm{SD}=516)$ (after presentation of third sentence at $6500 \mathrm{~ms}$ ) to respond to neutral, and $3497 \mathrm{~ms}$ $(\mathrm{SD}=538)$ to emotionally charged arguments. Accuracy scores for the neutral arguments were $65.9 \%(\mathrm{SD}=.8 .4)$ compared to $64.5 \%$ (SD $=6.2$ ) for the emotionally salient trials. The mean reaction times for the baseline condition were significantly lower $(1665 \mathrm{~ms}(\mathrm{SD}=403)$ and 1594 $(\mathrm{SD}=416)$ for emotional and neutral respectively), and accuracy scores significantly higher $(98 \%(\mathrm{SD}=.3 .8)$ and 98.1 ( $\mathrm{SD}=4.9$ ) for emotional and neutral respectively), than the reasoning trials. There were no significant differences between the two baselines. Subjects were not given feedback about their performance. The fMRI results are based on all trials.

Consistent with our previous studies (Goel et al., 2000; Goel and Dolan, 2003) the main effect of reasoning (neutral reasoning + emotional reasoning trials - neutral baseline - emotional baseline trials) resulted in activation of bilateral striate cortex (BA 17), lingual gyri (BA 18) $(-12,-84$, $2 ; Z=5.25$ and $16,-94,2 ; Z=4.96)$, left cerebellum (10, $-76,-30 ; Z=4.07)$, left middle temporal lobe (BA 21/22) $(-54,-46,0 ; Z=3.72)$, left temporal pole (BA 21/38) 
$(-50,8,-18 ; Z=4.60)$, right basal ganglia nuclei (accumbens, caudate nucleus, and putamen) $(12,8,-8 ; Z=$ $4.10)$, medial frontal gyrus (BA 6) $(0,10,60 ; Z=3.41)$, left middle frontal gyrus (BA 6) $(-38,2,58 ; Z=3.83)$, and left inferior frontal gyrus (BA 44) $(-56,18,26 ; Z=3.55)$.

Our primary interest in the present study was in the Content (salient and neutral) by Task (reasoning and baseline) interaction. Reasoning specific to neutral arguments [(neutral reasoning - neutral baseline) - (emotional reasoning - emotional baseline)] showed enhanced activation in left L/DLPFC (BA 44, 8) $(-46,6,32 ; \mathrm{Z}=4.62)$ (Fig. 2a and $2 b)$. Reasoning specific to emotional content [(emotional reasoning - emotional baseline) - (neutral reasoning - neutral baseline)] revealed activation in VMPFC $(-10$, $42,-16 ; Z=4.65$ and 6, 46, $-16 ; Z=4.59$ ) (Fig. 2c and $2 \mathrm{~d})$ and right fusiform gyrus (BA 37) $(24,-50,-10 ; Z=$ 4.46).

Consistent with these results the direct comparison of neutral with emotional reasoning showed enhanced activation of left lateral PFC during neutral reasoning $(-54,28$, $14 ; Z=3.66$ ). The reverse comparison (emotional reasoning - neutral reasoning) showed activation in $\operatorname{VMPFC}(0$, $26,-14 ; Z=3.66)$ and fusiform gyrus $(26,-54,-12 ; Z=$ 3.50 ). While these activations did not survive correction, the same comparisons for neutral and emotional baseline statements did not reveal any activation in L/DLPFC, VMPFC, or fusiform gyrus.

To further examine the influence of emotional saliency on the reasoning process, we examined how the neural response in L/DLPFC and VMPFC covaried with the subjects' postscan ratings of saliency of arguments. We constrained this analysis to regions where there was a significant interaction between reasoning and emotional saliency $\{[($ emotional reasoning - emotional baseline) - (neutral reasoning - neutral baseline $)],(P<0.05$, uncorrected $)\}$. A significant effect was evident indicating a positive covariation within VMPFC $(-10,42,-16 ; 10,34,-16)$ and the subjects' postscan ratings of saliency of arguments $(P<$ 0.05 , corrected) (Fig. 2e). The reverse comparison (masked inclusively by the interaction [(neutral reasoning - neutral baseline) - (emotional reasoning - emotional baseline)], $\mathrm{p}$ $<.05$, uncorrected) revealed left L/DLPFC (BA 8, 9, 47) $(-56,8,30 ;-46,30,24 ;-44,20,-4)$ negatively covaried with subjects' postscan ratings of saliency of arguments $(P$ $<0.05$, corrected) (Fig. 2f).

\section{Discussion}

The main effect of reasoning activated bilateral striate cortex (BA 17), lingual gyri (BA 18), left cerebellum, left middle temporal lobe (BA 21/22), left temporal pole (BA 21/38), medial frontal gyrus (BA 6), left middle frontal gyrus (BA 6), and left inferior frontal gyrus (BA 44). A similar network has been identified in previous studies of logical reasoning (Goel et al., 2000; Goel and Dolan, 2003;
Houde et al., 2000; Knauff et al., 2002). However, in the present study we are interested in the two-way interaction between Content (emotionally salient and neutral) and Task (reasoning and baseline). Reasoning with neutral content resulted in greater activation in L/DLPFC cortex (over and above the neutral baseline) than reasoning with emotionally salient content (over and above the emotionally salient baseline). Consistent with this role, the L/DLPFC has been implicated in a series of "executive" cognitive tasks including the WCST (Drewe, 1974; Stuss et al., 2000), Tower of London (Fincham et al., 2002; Rowe et al., 2001; Shallice, 1988), the Stroop task (Perret, 1974; Weekes and Zaidel, 1996), design fluency (Jones-Gotman and Milner, 1977), cognitive estimation (Smith and Milner, 1984), planning and design (Goel and Grafman, 2000; Goel et al., 1997), and tasks (like humor appreciation) requiring the "breaking of mental sets" (Goel and Dolan, 2001a; Shammi and Stuss, 1999).

The introduction of emotionally salient content into the reasoning task results in enhanced activation in VMPFC and fusiform gyrus. A number of studies have activated these structures in emotion regulation tasks (Davidson et al., 2000a, 2000b; Reiman et al., 1997). However, our results go one step further. They demonstrate greater activation in VMPFC in emotionally salient reasoning (over and above the emotionally salient baseline) than neutral reasoning (over and above the neutral baseline). This interaction analysis, and the simple comparisons between salient and neutral reasoning trials (VMPFC activation), and salient baseline and neutral baseline trials (no VMPFC activation), suggest that VMPFC activation in our case cannot be explained simply by the presence of emotional saliency, and lead us to the conclusion that VMPFC is engaged by the reasoning process in the presence of emotional saliency.

There is one other potential interpretation of our results. Given the pattern of parameter estimates in Fig. 2e, it is possible that the "activation" in VMPFC during emotionally salient trials is actually "less" deactivation. It has been suggested that deactivation in VMPFC (along with other medial structures) during complex cognitive processing engaging DLPFC reflects a default mode of brain operation (Gusnard et al., 2001; Raichle et al., 2001). On this interpretation the "deactivation" in VMPFC during neutral reasoning trials reflects a baseline level of activity, given activation of DLPFC. The activation or "less" deactivation in VMPFC during emotionally salient trials may reflect an interruption in the baseline state, given decreased activation in DLPFC. This explanation, however, assumes that emotional reasoning trials involve less effort than neutral reasoning trials (hence decreased activation in DLPFC). This is not the case. The stimuli in the neutral and emotional conditions were matched for task difficulty and subjects' reaction times and accuracy scores confirm that the matching was successful. Therefore, our reciprocal activation in DLPFC and VMPFC in response to the emotional saliency of the reasoning material cannot be explained simply as a 


\section{Reciprocal Activation in LPFC \& VMPFC in Response to Cold and Hot Reasoning}

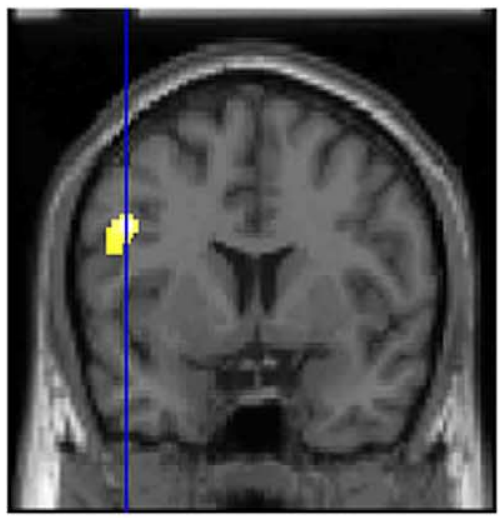

a

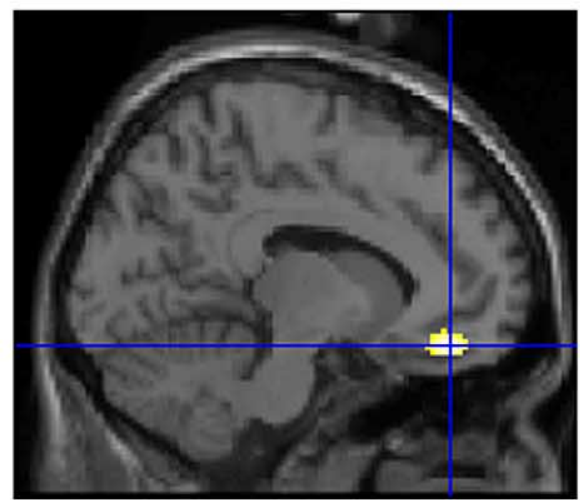

c

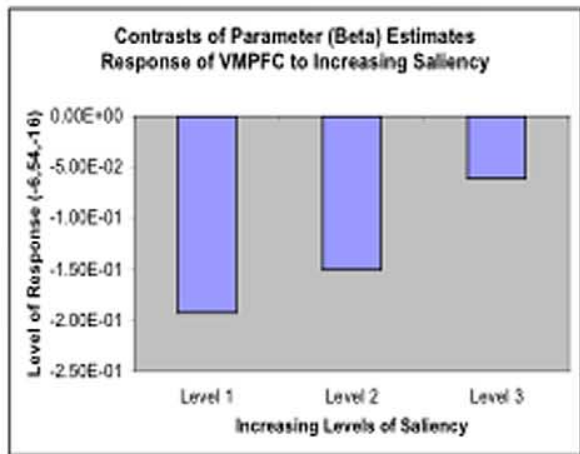

e

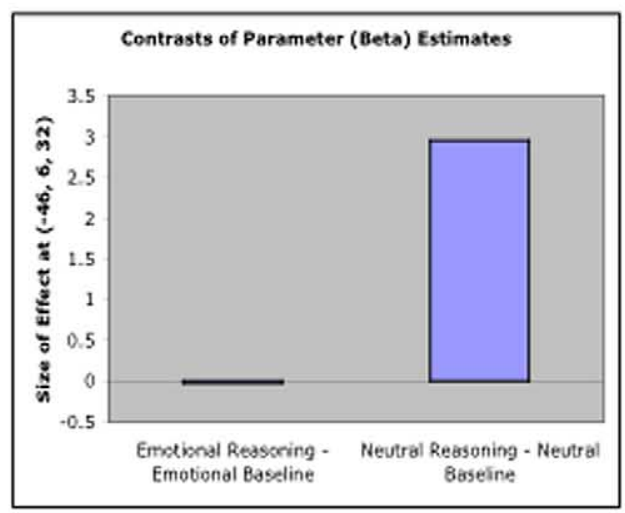

b

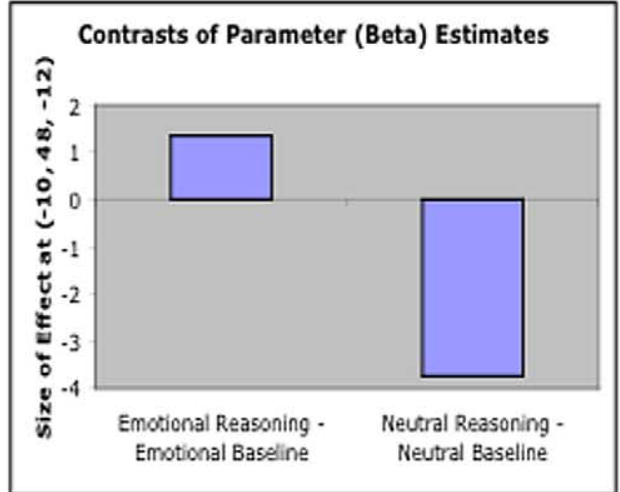

d

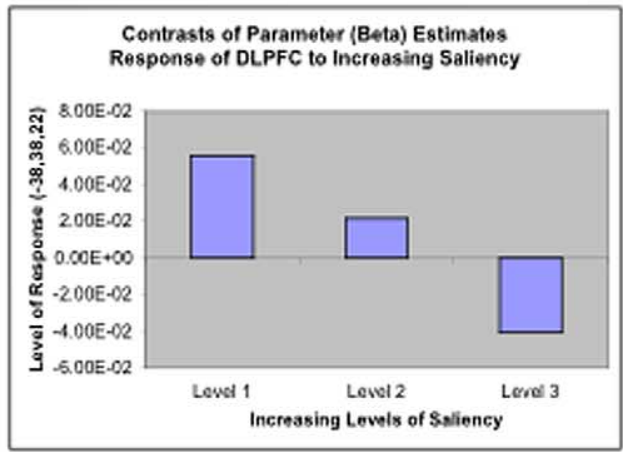

f

Fig. 2. Statistical parametric map (SPM) rendered into standard stereotactic space and superimposed on to coronal (a) and sagittal (c) sections of a magnetic resonance image (MRI) which is itself in standard space. (a) Modulation of the reasoning task from salient content to neutral content [(neutral reasoning neutral baseline) - (emotional reasoning - emotional baseline)] revealed activation in left inferior/middle frontal gyri $(\mathrm{BA} 44,8)(-46,6,32 ; Z=4.62)$. (b) Condition-specific parameter (beta) estimates show that the L/DLPFC is more activated during neutral reasoning trials than during emotionally salient reasoning trials. (c) The reverse modulation of reasoning from neutral content to emotionally salient content [(emotional reasoning - emotional baseline) - (neutral reasoning - neutral baseline)] resulted in activation of bilateral VMPFC $(-10,42,-16 ; Z=4.65 \& 6,46,-16 ; Z=4.59)$ (BA 11/25) and right fusiform gyrus (BA 37) $(24,-50,-10 ; Z=4.46)$ (not shown). (d) Condition-specific parameter (beta) estimates show that the VMPFC is more functionally deactivated during neutral reasoning trials than during emotionally salient reasoning trials. (e) Parameter (beta) estimates showing increasing activity in VMPFC as a function of increasing levels of emotional saliency of content (from level 1 to level 3) (single subject). (f) Parameter (beta) estimates showing decreasing activity in L/DLPFC as a function of increasing levels of emotional saliency of content (single subject). 
default mode of brain operation. It is real effect of the experimental manipulation.

While we are unaware of other studies explicitly manipulating emotional saliency of content in reasoning or decision-making tasks, our findings are consistent with several related fMRI studies involving "personal judgments." One recent study on logical reasoning demonstrated activation in VMPFC when the logical response was overcome by belief bias (i.e., when subjects responded on the basis of personal beliefs rather than the logic of the situation) (Goel and Dolan, 2003). A study involving moral personal, moral impersonal, and nonmoral judgments has shown greater activation of MPFC in the moral personal condition compared to the other conditions (Greene et al., 2001). ${ }^{3}$ Our results are also consistent with neuroanatomical evidence. The VMPFC receives projections from the amygdala and medial temporal and thalamic structures and in turn is robustly interconnected to the lateral prefrontal cortex (Barbas, 2000). It is well situated to serve the role we postulate for it.

In summary, while one or two studies have recently looked at the interaction of emotion and cognition in a dual-task paradigm, by manipulating subject mood with stimuli unrelated to the cognitive task (Gray et al., 2002), we believe this is the first study to use a reasoning task and directly manipulate the emotional saliency of the content of reasoning material in a single-task paradigm. Our findings indicate that neural systems engaged by reasoning tasks are dynamically configured as a function of the emotional saliency of the content. Specifically, we show a reciprocal response in L/DLPFC and VMPFC that reflects the degree to which reasoning is "cold" or "hot."

\section{Acknowledgments}

V.G. is supported by a McDonnell-Pew Program in Cognitive Neuroscience Award, Premier's Research Excellence Award, and National Science and Engineering Research Council of Canada and Canadian Institutes of Health Research grants. R.J.D. is supported by a Wellcome Trust Programme Grant.

\section{References}

Anderson, S.W., Damasio, H., Tranel, D., Damasio, A.R., 2000. Long-term sequelae of prefrontal cortex damage acquired in early childhood. Dev. Neuropsychol. 18 (3), 281-296.

Ashburner, J., Friston, K.J., 1999. Nonlinear spatial normalization using basis functions. Human Brain Mapp. 7 (4), 254-266.

\footnotetext{
${ }^{3}$ One study that seems to be at odds with our results has reported the left DLPFC to be the site of integration of emotion and cognition (Gray et al., 2002). However, the comparison may be misleading. This study used a working memory task instead of a reasoning task and manipulated emotions/mood independently of the cognitive task.
}

Barbas, H., 2000. Connections underlying the synthesis of cognition, memory, and emotion in primate prefrontal cortices. Brain Res. Bull. 52 (5), 319-330.

Bechara, A., Damasio, H., Damasio, A.R., 2000. Emotion, decision making and the orbitofrontal cortex. Cereb. Cortex 10 (3), 295-307.

Bradley, M.M., Lang, P.J., 1994. Measuring emotion: the Self-Assessment Manikin and the Semantic Differential. J. Behav. Ther. Exp. Psychiatry 25 (1), 49-59.

Damasio, A.R., 1996. The somatic marker hypothesis and the possible functions of the prefrontal cortex. Phil. Trans. R. Soc. Lond. B. Biol. Sci. 351 (1346), 1413-1420.

Damasio, A.R., 1997. Neuropsychology. Towards a neuropathology of emotion and mood. Nature 386 (6627), 769-770.

Davidson, R.J., Jackson, D.C., Kalin, N.H., 2000a. Emotion, plasticity, context, and regulation: perspectives from affective neuroscience. Psychol. Bull. 126 (6), 890-909.

Davidson, R.J., Putnam, K.M., Larson, C.L., 2000b. Dysfunction in the neural circuitry of emotion regulation-a possible prelude to violence. Science 289 (5479), 591-594.

Deichmann, R., Josephs, O., Hutton, C., Corfield, D.R., Turner, R., 2002. Compensation of susceptibility-induced BOLD sensitivity losses in echo-planar fMRI imaging. NeuroImage 15 (1), 120-135.

Drewe, E.A., 1974. The effect of type and area of brain lesion on Wisconsin Card Sorting Test performance. Cortex 10, 159-170.

Evans, A.C., Collins, D.L., Mills, S.R., Brown, E.D., Kelly, R.L., Peters, T.M., 1993a. 3D statistical neuroanatomical models from 305 MRI volumes, in: Proceedings. IEEE-Nuclear Science Symposium and Medical Imaging Conference, pp. 1813-1817.

Evans, J.S.B.T., Newstead, S.E., Byrne, R.M.J., 1993b. Human Reasoning: The Psychology of Deduction. Erlbaum, Hillsdale, NJ.

Fincham, J.M., Carter, C.S., van Veen, V., Stenger, V.A., Anderson, J.R., 2002. Neural mechanisms of planning: a computational analysis using event-related fMRI. Proc. Natl. Acad. Sci. USA 99 (5), 3346-3351.

Friston, K.J., Holmes, A.P., Worsley, K.J., Poline, J.B., Frith, C.D., Frackowiak, R.S.J., 1995. Statistical parametric maps in functional imaging: a general approach. Human Brain Mapp. 2, 189-210.

Genovese, C.R., Lazar, N.A., Nichols, T., 2002. Thresholding of statistical maps in functional neuroimaging using the false discovery rate. NeuroImage 15 (4), 870-878.

Goel, V., Buchel, C., Frith, C., Dolan, R.J., 2000. Dissociation of mechanisms underlying syllogistic reasoning. NeuroImage 12 (5), 504-514.

Goel, V., Dolan, R.J., 2001a. Functional neuroanatomy of humor: segregating cognitive and affective components. Nature Neurosci. 4 (3).

Goel, V., Dolan, R.J., 2001b. Functional neuroanatomy of three-term relational reasoning. Neuropsychologia 39 (9), 901-909.

Goel, V., Dolan, R.J., 2003. Explaining modulation of reasoning by belief. Cognition 87 (1), B11-22.

Goel, V., Gold, B., Kapur, S., Houle, S., 1997. The seats of reason: a localization study of deductive and inductive reasoning using PET (O15) blood flow technique. NeuroReport 8 (5), 1305-1310.

Goel, V., Gold, B., Kapur, S., Houle, S., 1998. Neuroanatomical correlates of human reasoning. J. Cogn. Neurosci. 10 (3), 293-302.

Goel, V., Grafman, J., 2000. The role of the right prefrontal cortex in Ill-structured problem solving. Cogn. Neuropsychol. 17 (5), 415-436.

Goel, V., Grafman, J., Tajik, J., Gana, S., Danto, D., 1997. A study of the performance of patients with frontal lobe lesions in a financial planning task. Brain 120, 1805-1822.

Goldman-Rakic, P.S., 1987. Circuitry of primate prefrontal cortex and regulation of behavior by representational memory, in: Mountcastle, V.B., Plum, F., Geiger, S.R. (Eds.), Handbook of Physiology: The Nervous System, Vol. 5, Higher Functions of the Brain, Part 1, American Physiological Society, Washington, DC, pp. 373-417.

Gorno-Tempini, M.L., Hutton, C., Josephs, O., Deichmann, R., Price, C., Turner, R., 2002. Echo time dependence of BOLD contrast and susceptibility artifacts. NeuroImage 15 (1), 136-142. 
Gray, J.R., Braver, T.S., Raichle, M.E., 2002. Integration of emotion and cognition in the lateral prefrontal cortex. Proc. Natl. Acad. Sci. USA 99 (6), 4115-4120.

Greene, J.D., Sommerville, R.B., Nystrom, L.E., Darley, J.M., Cohen, J.D., 2001. An fMRI investigation of emotional engagement in moral judgment. Science 293 (5537), 2105-2108.

Gusnard, D.A., Akbudak, E., Shulman, G.L., Raichle, M.E., 2001. Medial prefrontal cortex and self-referential mental activity: relation to a default mode of brain function. Proc. Natl. Acad. Sci. USA 98 (7), 4259-4264.

Hariri, A.R., Bookheimer, S.Y., Mazziotta, J.C., 2000. Modulating emotional responses: effects of a neocortical network on the limbic system. NeuroReport 11 (1), 43-48.

Harlow, J.M., 1868. Recovery after severe injury to the head. Publ. Massachusetts Med. Soc. 2, 327-346.

Henle, M., 1962. On the relation between logic and thinking. Psychologic. Rev. 69 (4), 366-378.

Houde, O., Zago, L., Crivello, F., Moutier, S., Pineau, A., Mazoyer, B., Tzourio-Mazoyer, N., 2001. Access to deductive logic depends on a right ventromedial prefrontal area devoted to emotion and feeling: evidence from a training paradigm. NeuroImage 14 (6), 1486-1492.

Houde, O., Zago, L., Mellet, E., Moutier, S., Pineau, A., Mazoyer, B., Tzourio-Mazoyer, N., 2000. Shifting from the perceptual brain to the logical brain: the neural impact of cognitive inhibition training. J. Cogn. Neurosci. 12 (5), 721-728.

Hutton, C., Bork, A., Josephs, O., Deichmann, R., Ashburner, J., Turner, R., 2002. Image distortion correction in fMRI: a quantitative evaluation. NeuroImage 16 (1), 217-240.

Jones-Gotman, M., Milner, B., 1977. Design fluency: the invention of nonsense drawings after focal cortical lesions. Neuropsychologia 15 (4, 5), 653-674.

Knauff, M., Mulack, T., Kassubek, J., Salih, H.R., Greenlee, M.W., 2002. Spatial imagery in deductive reasoning: a functional MRI study. Brain Res. Cogn. Brain Res. 13 (2), 203-212.

Koechlin, E., Corrado, G., Pietrini, P., Grafman, J., 2000. Dissociating the role of the medial and lateral anterior prefrontal cortex in human planning. Proc. Natl. Acad. Sci. USA 97 (13), 7651-7656.

Lane, R.D., Reiman, E.M., Ahern, G.L., Schwartz, G.E., Davidson, R.J., 1997. Neuroanatomical correlates of happiness, sadness, and disgust. Am. J. Psychiatry 154 (7), 926-933.
Parsons, L.M., Osherson, D., 2001. New evidence for distinct right and left brain systems for deductive versus probabilistic reasoning. Cereb. Cortex 11 (10), 954-965.

Perret, E., 1974. The left frontal lobe of man and the suppression of habitual responses in verbal categorical behaviour. Neuropsychologia 12, 323-374.

Pietrini, P., Guazzelli, M., Basso, G., Jaffe, K., Grafman, J., 2000. Neural correlates of imaginal aggressive behavior assessed by positron emission tomography in healthy subjects. Am. J. Psychiatry 157 (11), 1772-1781.

Raichle, M.E., MacLeod, A.M., Snyder, A.Z., Powers, W.J., Gusnard, D.A., Shulman, G.L., 2001. A default mode of brain function. Proc. Natl. Acad. Sci. USA 98 (2), 676-682.

Reiman, E.M., Lane, R.D., Ahern, G.L., Schwartz, G.E., Davidson, R.J., Friston, K.J., Yun, L.S., Chen, K., 1997. Neuroanatomical correlates of externally and internally generated human emotion. Am. J. Psychiatry 154 (7), 918-925.

Rowe, J.B., Owen, A.M., Johnsrude, I.S., Passingham, R.E., 2001. Imaging the mental components of a planning task. Neuropsychologia 39 (3), 315-327.

Shallice, T., 1988. From Neuropsychology to Mental Structure. Cambridge University Press, Cambridge.

Shammi, P., Stuss, D.T., 1999. Humour appreciation: a role of the right frontal lobe. Brain 122 (Pt 4), 657-666.

Smith, M.L., Milner, B., 1984. Differential effects of frontal-lobe lesions on cognitive estimation and spatial memory. Neuropsychologia 22 (6), 697-705.

Stuss, D.T., Levine, B., 2002. Adult clinical neuropsychology: lessons from studies of the frontal lobes. Annu. Rev. Psychol. 53, 401-433.

Stuss, D.T., Levine, B., Alexander, M.P., Hong, J., Palumbo, C., Hamer, L., Murphy, K.J., Izukawa, D., 2000. Wisconsin Card Sorting test performance in patients with focal frontal and posterior brain damage: effects of lesion location and test structure on separable cognitive processes. Neuropsychologia 38 (4), 388-402.

Weekes, N.Y., Zaidel, E., 1996. The effects of procedural variations on lateralized Stroop effects. Brain Cogn. 31 (3), 308-330.

Worsley, K.J., Friston, K.J., 1995. Analysis of fMRI time-series revisited-again. NeuroImage 2, 173-181. 